\begin{tabular}{ccc}
\hline & International Journal of Engineering \& Technology, $7(4.38)(2018) 1125-1127$ \\
SPC & International Journal of Engineering \& Technology \\
Website $:$ www.sciencepubco.com/index.php/IJET \\
Research paper
\end{tabular}

\title{
Evaluation Method of the Fatigue Life of Power Plant Pipelines
}

\author{
Natalya Y. Golovina ${ }^{1}$, Svetlana Y. Krivosheeva ${ }^{2}$ \\ ${ }^{1}$ Associate Professor, Ph.D.,Tyumen Industrial University, branch in Surgut, Surgut, Russia (628400, Surgut, Entuziastov str., 38), e- \\ ${ }^{2}$ Ph.D. Tyumen Industrial University, branch in Surgut, Surgut, Russia (628400, Surgut, Entuziastov str., 38) \\ E-mail: ntgolovina@ rambler.ru
}

\begin{abstract}
The method for estimating the fatigue life of power plant pipelines based on the results of one or two tests without destruction is proposed. The proposed method uses the classical model of fatigue resistance. Weller curves for power plant pipelines are determined experimentally, the experimental data are presented. Three curves are constructed for the probability of destruction of $2 \%, 50 \%$, and $98 \%$. The proposed method uses the hypothesis of linear summation of Palmgren-Miner injuries. When developing the method, much attention is paid to the ways of schematizing the actual process of loading the pipelines. Calculation of the distribution of the total number of cycles was carried out by the "Rainflow" method and the Gusev method. The application of this method with a set of design measures provides more than fivefold margin for fatigue resistance of all pipelines of the power plant.
\end{abstract}

Keywords: fatigue / longevity / pipeline. Nomenclature: $\mathrm{kHz}$-kilohertz $\mathrm{kgf} / \mathrm{mm}^{2}$ - kilogram-force/square millimetre

\section{Introduction}

One of the important problems of the operation of power plants for a long service life is the provision of a stock of fatigue resistance of pipelines $[8,12-16]$. At the same time, it is necessary to solve the problem of operative assessment of the fatigue life of the power plant pipelines based on the results of one or two tests without destruction. Since the evaluation of the resource after the destruction of the pipeline requires considerable time and resources for testing.

The most reliable methods for assessing fatigue life are methods that consider the actual loading processes of pipelines during the tests and their fatigue properties [4-7]. This method of estimating the longevity of pipelines has been created and is widely used for their development for a long service life. In this article, a brief description of this method is given and an example illustrating its effectiveness is considered.

\section{Materials and Methods}

The proposed method uses a fatigue resistance model based on the Weller curve $[1-3,10,22]$. It is assumed that the loading is performed by cycles with a constant amplitude according to the harmonic law. During the tests, the Weller curves are usually constructed with an equal probability of destruction [11,21,23].

Figure 1 shows set of three curves for the probability of destruction of $2 \%, 50 \%$, and $98 \%$.

Numerous experimental data show that the $\sigma-N$ dependence in region 2 has the form:

$\sigma^{m} \cdot N=C$,

where $\sigma$ - amplitude of the cycle stresses; $N$ - number of cycles to failure; $m$ and $C$ - constants characterizing the material, the geometry of the part, manufacturing technology, etc.

The range of $m$ values for metals is usually $3 \leqslant m \leqslant 10$.

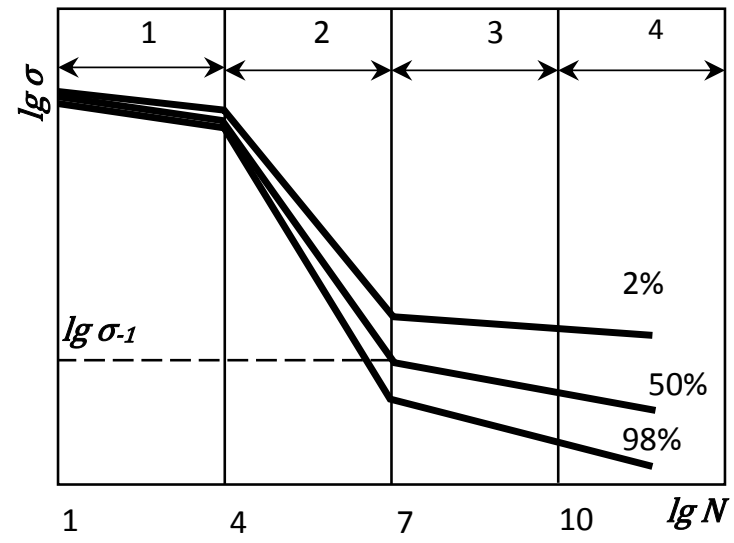

Fig. 1: Type of Weller curve for pipeline materils.

Experimental data on the nature of the dependence in region 3 remained under-represented in the literature, since their preparation is a very laborious task. It is usually assumed that there is some stress $\sigma_{-l}$, and when loaded with an amplitude $\sigma \leqslant \sigma_{-l}$, the number of cycles before destruction tends to infinity [17-19].

To evaluate the service life of power plant pipelines, Weller curves were experimentally determined on samples manufactured using standard technology. The samples were subjected to flexural loading, since in all cases of fatigue destruction of pipelines of power plants, the structure of the crack development zone, characteristic for bending, is noted. The experimental data obtained for the three types of pipelines are given in Table 1 for Weller curves corresponding to the probability of destruction of $2 \%$ and $50 \%$ at four points. The values for $N=10^{7}$ и $10^{10}$ were obtained by interpolation of the curves in the range $10^{4} \leqslant N \leqslant 10^{6}[1]$.

The developed method uses the Palmgren-Miner linear damage hypothesis $[9,25]$. It is assumed that when the sample is loaded with cycles with different amplitudes, the fractions of damage from the 
action of individual cycles are summed linearly: $D_{\Sigma}=D_{1}+D_{2}+$ $D_{3}+\cdots+D_{n}=\sum_{i} D_{i}$,

where $D_{\Sigma}$ - total damage; $D_{i}$ - percentage of damage caused by cycles with amplitude $\sigma_{\mathrm{i}}$.

The failure conditions for this hypothesis are written as:

$\Sigma_{i} \cdot D_{i}=1$,

$D_{i}$ is calculated by the formula:

$D_{i}=\frac{n\left(\sigma_{i}\right)}{N\left(\sigma_{i}\right)}$,

where $n\left(\sigma_{i}\right)$ - total number of cycles with amplitude $\sigma_{i} ; N\left(\sigma_{i}\right)$ - the number of cycles with amplitude $\sigma_{i}$, required for destruction (Weller curve).

Loading of the pipelines of the power plant during the tests is random. Therefore, the processes of training and fatigue softening noted in [7], characteristic for block loading, are extremely weak and do not have a great influence on the accuracy of the results obtained. This circumstance justifies the choice of the linear hypothesis.

Table 1: Dependence of the cyclic life $N$ from the material and stress $\sigma$ $\left(\mathrm{kgf} / \mathrm{mm}^{2}\right)$

\begin{tabular}{|c|c|c|c|c|c|}
\hline \multirow{2}{*}{ Material } & \multirow{2}{*}{$\mathrm{P}, \%$} & \multicolumn{4}{|c|}{ Cyclic life $N$} \\
\cline { 3 - 6 } & & $10^{4}$ & $10^{6}$ & $10^{7}$ & $10^{10}$ \\
\hline \multirow{2}{*}{ 12XI8HIOT } & 2 & 39,8 & 13,4 & 7,9 & 6,3 \\
& 50 & 69,2 & 22,9 & 13,1 & 10,9 \\
\hline \multirow{2}{*}{ VNS - 25 (BHC-25) } & 2 & 30,1 & 9,5 & 5,3 & 4,3 \\
& 50 & 56,2 & 20,0 & 9,8 & 7,9 \\
\hline \multirow{2}{*}{ JeI-811 (ЭИ - 811) } & 2 & 41,6 & 12,3 & 6,7 & 5,6 \\
& 50 & 72,4 & 22,9 & 12,6 & 10,4 \\
\hline
\end{tabular}

As the value of $D \Sigma$ approaches unity, the probability of destruction of this pipeline increases, and at $D_{\Sigma}=1$ it reaches a value equal to the probability of destruction for the Weller curve used in the calculation.

When developing the method, great attention was paid to the ways of schematization of the real process of loading the pipes $\sigma(t)$, i.e. methods of partitioning a random process into a set of individual load cycles with different amplitudes and thus obtaining the distribution $n\left(\sigma_{i}\right)$. At present, the most accurate method is the method of complete cycles [10,13,14]. In this paper, its modified version was used - the "Rainflow" method, which is associated with the need for digital processing of large amounts of information that enter the computer in real time with a frequency of up to $4 \mathrm{kHz}$. The complexity of the hardware processing of information flow of this intensity limits the possibilities for widespread adoption of this method.

In this connection, various analytical methods for finding the distribution $n\left(\sigma_{i}\right)$, based on the assumption of a random Gaussian character of the loading process, were considered. There are approaches $[9,20]$ based on various methods of schematization of the process, for example, Rice's solution, the method for swing, and others. They use statistical characteristics of loading processes, such as the power spectrum, the correlation function of the process, etc.

The Gusev method $[9,20,24]$, which is closest to the "Rainflow" method, was chosen, according to which:

$n\left(\sigma_{i}\right)=f\left(\sigma_{i}\right) \cdot d \sigma \cdot \bar{N}_{\text {max }}$

where $\bar{N}_{\text {max }}$ - the average number of maxima per unit time; $f\left(\sigma_{i}\right)$ - functions of the density distribution of the amplitudes of cycles; $d \sigma$ - sampling step;

$$
\begin{aligned}
& f(\sigma)=\left\{\begin{array}{lr}
K^{2} \sigma \exp \left(-K^{2} d^{2} \frac{\sigma^{2}}{2}\right), & 0 \leq \sigma \leq \sigma_{*} \\
K^{-1} c \sigma \exp \left(\frac{-\sigma^{2}}{2}\right), & \sigma \geq \sigma_{*}
\end{array}\right. \\
& \alpha^{2}=2 \frac{a+3 b}{a b}, \\
& C=K^{-\frac{a}{b}} \exp \left(\frac{\sigma_{*}^{2}}{2}\right),
\end{aligned}
$$

$\sigma_{*}=\frac{\sqrt{a \ln K}}{K}$

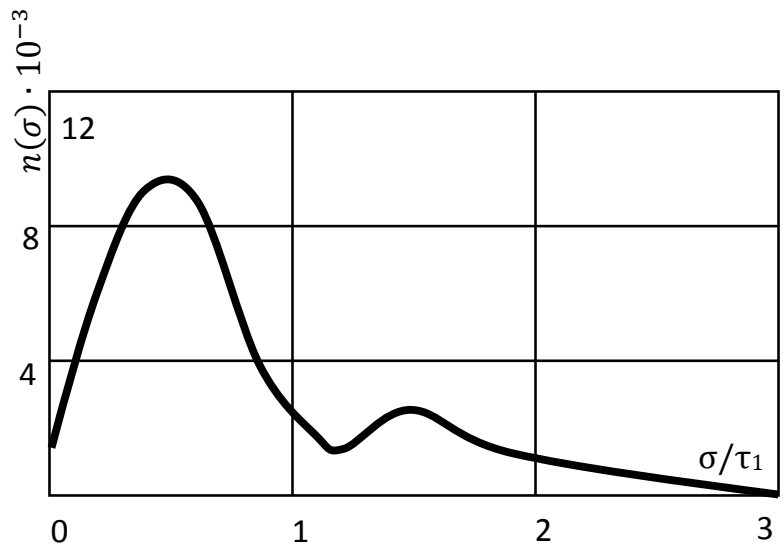

Fig. 2: Distribution of the number of amplitude loading cycles for power lines

Parameters $a$ and $b$ can be found from the solution of the transcendental system of equations given in [9], where tables of values of $a$ and $b$ are also given depending on the parameter $K$, which characterizes the complexity of the process, i.e. the ratio of the number of extrema in it to the number of zeros. The values of $K$ and $\bar{N}_{\text {max }}$ can be expressed by the spectrum of the process:

$K=\frac{\tau_{1} \tau_{3}}{\tau_{2}^{2}}$

$\bar{N}_{\text {max }}=\frac{\tau_{3}}{2 \pi \tau_{2}}$

where:

$\tau_{1}^{2}=\frac{1}{2 \pi} \int_{0}^{\infty} S(\omega) d \omega$

$\tau_{2}^{2}=\frac{1}{2 \pi} \int_{0}^{\infty} \omega^{2} S(\omega) d \omega$

$\tau_{3}^{2}=\frac{1}{2 \pi} \int_{0}^{\infty} \omega^{4} S(\omega) d \omega$

$S(\omega)$ - process spectrum.

In (1), it was assumed that $d \sigma=0,1 \tau_{l}$, since model calculations for different values of $K$ and $S(\omega)$ showed that a further decrease of do would not lead to an increase in accuracy.

The problem of determining $S(\omega)$ for stationary stochastic processes is considered in detail, for example, in [17].

The equivalent load model was used to account for the effect of static stress $\sigma_{m}$ on the service life of pipelines in power plants. Calculation of it was carried out according to the formula:

$\sigma_{\text {equiv }}=\sigma_{a}+\psi_{a} m$

where $\psi$ - static load influence factor.

In the calculations, the value $\psi=0,1$ was chosen, which is the most characteristic for pipeline materials. In this method, the transition to an equivalent load was carried out in the Weller curve, i.e. the total damageability was calculated by the formula:

$D_{\Sigma}=\sum_{i} \frac{n\left(\sigma_{i}\right)}{N\left(\sigma_{i \text { equiv }}\right)}$

where $\sigma_{i \text { equiv }}=\sigma_{i}+\psi \sigma_{m}$

The value of $\sigma_{m}$ was measured. In the absence of measurements, the maximum permissible value $\sigma_{m}$ for the mounting voltages should be taken. In some cases, a series of calculations were performed with the values: $\sigma_{m}=0 ; 7 ; 20 ; 50 \mathrm{kgf} / \mathrm{mm}^{2}$.

\section{Results}


This approach was used to evaluate the pipeline operation life based on the results of one or two tests without destruction. In this case, the calculation of the distribution $n\left(\sigma_{i}\right)$ was carried out both by the "Rainflow" method and by the Gusev method. The obtained results show that the $n\left(\sigma_{i}\right)$ distributions found by the two methods coincide both in the form of the function and in its numerical characteristics. The discrepancy for typical loading processes does not exceed $20 \%$ in amplitude. A typical distribution of $n\left(\sigma_{i}\right)$ for stresses in the pipelines of the power plant is shown in Fig. 2

The nonmonotonicity of the curve depicted on it is explained by the fact that the schematization methods used are based on the sequential separation of cycles with the lowest amplitude from the process. As the amplitude of the selected cycles increases, the broadband factor of the process changes (first $K>1$, then $K \approx 1$ ). The distribution of the cycle amplitudes for the two indicated zones of the values of $\mathrm{K}$ is different, which gives rise to a nonmonotonicity of the curve.

For the obtained distributions $n\left(\sigma_{i}\right)$, the value of $D_{\Sigma}$ was calculated for one test of power plants. Statistics of fatigue failure of the pipelines of the power plant showed that the value of $D_{\Sigma}$ at the moment of failure is $0.6 \quad \ldots 1.2$ for the Weller curve of $50 \%$ probability, which makes it possible to reliably isolate those pipelines in the design of power plants whose resource availability is insufficient.

A characteristic example of using the described method to refine the structure for the required resource is the design development of one of the power plant pipelines.

In the original design, the life of this pipeline did not exceed two tests. In this case, the value of $D_{\Sigma}$ calculated by the "Rainflow" method for one test is $0.15 \ldots 0.55$, depending on the cyclogram of the operation of the power plant for a $50 \%$ fracture probability $\left(\sigma_{m}\right.$ $=7 \mathrm{kgf} / \mathrm{mm}^{2}$ ). The tests for which resonance excitation of the pipeline oscillations were observed corresponded to large values of $D_{\Sigma}$. The change in design reduced the quality of the resonant oscillations, and the value of $D_{\Sigma}$ turned out to be in the range of 0.05 ... 0.4. At the same time, the destruction of this pipeline was also observed, but the resource of the work slightly increased and amounted to two or three tests.

Further modification of the design consisted in the transition to another grade of pipe. The value of $D_{\Sigma}$ for one test for this version was $0.01 \ldots 0.026$. At the same time, pipeline destruction did not occur during the four tests, but the achieved value of $D_{\Sigma}$ indicated a real possibility of fatigue failure of the pipeline for twenty trials or more. To guarantee the resource in twenty trials, the fourth variant of the pipeline design was introduced, for which $D_{\Sigma}$ was $0.003 \ldots 0.007$ per one test, which indicates a sufficient margin of fatigue life of the pipeline for a given resource.

Further experience in the operation of the power plant showed that in the range of 10 or more tests fatigue failure of the pipeline is not observed.

Thus, the use of this method with a set of design measures allowed to provide more than fivefold margin for fatigue resistance of all the pipelines of the power plant.

\section{References}

[1] A.V. Aleksandrov, Resistance of materials: 2nd edition, Higher Education, Moscow, 2000, p. 560.

[2] K. Bate, E. Wilson, Numerical Analysis Methods and the Finite Element Method, Stroyizdat, Moscow, 1982, p. 448.

[3] V.V. Bolotin, Methods of probability theory and reliability theory in the calculation of structures, Stroiizdat, Moscow, 1982, p.351.

[4] N.Y. Golovina, Questions of the stability of forced transverse vibrations of flexible metal pipelines, Scientific Review 10 (2014) 6366.

[5] N.Y. Golovina, A New Approach to the Study of Forced Transverse Vibrations of Flexible Metal Pipelines for Stability, Izvestiva Vysshikh Uchebnykh Zavedenii. Oil and gas 1 (2005) 70-74.
[6] N. Y. Golovina, S. Y. Krivosheeva, Research in area of longevity of sylphon scraies, IOP Conf. Series: Earth and Environmental Science 115 (2018)

[7] N.Y. Golovina, S.V. Krivosheyeva, Investigation of various factor influencing the vibrational strength of flexible metal pipelines, Natural and technical sciences 6 (2015) 269-272.

[8] E.V. Dedikov, V.A. Markelov, G.S. Klishin, Calculation of the strength of technological pipelines of the compressor station, The gas industry 8 (1999) 31-33.

[9] O.A. Zakirov, A.V. Greb, M.A. Shatalina, A.Kh. Gabbasova Calculation of technological pipelines as spatial structures considering the energy of elastic deformation, Preprint Ufa: UGNTU 7 (1999) p. 32

[10] V.P. Kogaev, Calculations for strength at stresses, variables in time, Mashinovedenie, Moscow, 1987, p. 232.

[11] N.S. Kuznetsov, P.S. Tarasyuk, Determination of defects in the wall of the operating pipeline by a vibroacoustic method, Issues in the theory and practice of control and diagnostics 7 (2000) 5-7.

[12] V.Y. Magalif, The program of calculation of pipelines on computers, Methods and techniques of modern design 6 (1970).

[13] G.I. Marchuk, Methods of computational mathematics, Nauka, Moscow, 1977

[14] V.Z. Parton, Mechanics of destruction: From theory to practice, Nauka, Moscow, 1990, p. 240.

[15] E.V. Poznyak, Yu.N. Samogin, V.E Khromatov, Calculation of the spectrum of natural frequencies of equipment and pipelines of the reactor installation, Dynamics, strength and wear resistance of machines 4 (1998) 40-43.

[16] Yu.N. Samogin, V.P. Chirkov, Restoration of the vibration field of structures according to the indications of vibration probes, Dynamics, strength and wear resistance of machines 4 (1998) 31-34.

[17] A.F. Selikhov, I.G. Khlebnikov, The method of statistical estimation of the threshold of sensitivity of fatigue life, Nauka, Moscow, 1969.

[18] S.V. Serensen, V.P. Kogaev, R.M. Shnederovich, Bearing capacity and calculation of machine parts for durability, Mashgiz, Moscow, 1963.

[19] M. Shiratori, T. Miyoshi, X. Matsushita, Computational Mechanics of Destruction, Mir, Moscow, 1986, p.334.

[20] M.N. Stepanov, Statistical processing of the results of mechanical tests, Mechanical Engineering, Moscow, 1972.

[21] M.N. Stepanov, E.V. Hyacintov, Tiredness of light structural alloys, Mechanical Engineering, Moscow, 1973.

[22] K. Hellan, Introduction to the mechanics of destruction, Mir Moscow, 1988, p. 364.

[23] Accidents on pipeline transport: Based on the materials of the Ministry of Emergency Situations of Russia, Pipelines and ecology 4 (1998) 27.

[24] V.V. Bolotina, Oscillations of Linear Systems, Mashinostroenie, Moscow, 1979, p. 352.

[25] V.P. Kogaev, N.A. Makhutov, A.P. Gusenkov, Methods for calculating the characteristics of fatigue resistance, Mashinovedenie, Moscow, 1982, p. 80. 